\title{
Using a linear mixed-effect model framework to estimate multivariate generalizability theory parameters in $\mathrm{R}$
}

\author{
Zhehan Jiang $^{1} \cdot$ Mark Raymond $^{2} \cdot$ Dexin Shi $^{3} \cdot$ Christine DiStefano $^{4}$ \\ Published online: 15 May 2020 \\ (C) The Psychonomic Society, Inc. 2020
}

\begin{abstract}
Multivariate generalizability theory (mG-theory) is an important framework in many behavioral and educational studies, as it describes useful psychometric properties of multidimensional assessments. Nevertheless, the use of mG-theory estimation is limited due to the lack of available software for carrying out the necessary calculations: users rely heavily on independent software programs such as mGENOVA and the BUGS/JAGS suite of programs. Considering the prevalence of R software, this paper presents a solution using the glmmTMB package to accomplish the estimation task. Users adopting the proposed method may find it more convenient for conducting both applied investigation and simulation studies without the need to switch between different software programs.
\end{abstract}

Keywords Generalizability $\cdot$ Multivariate $\cdot \mathrm{R} \cdot \operatorname{lme} 4 \cdot \operatorname{glmmTMB} \cdot$ Mixed-model

\section{Introduction}

Since its introduction by Cronbach and colleagues in the 1960s, generalizability theory (G-theory) has played a critical role in research on behavioral and educational assessments (Cronbach, Gleser, \& Rajaratnam, 1963; Cronbach, Gleser, Nanda, \& Rajaratnam, 1972; Gleser, Cronbach, \& Rajaratnam, 1965). G-Theory extends classical test theory by using ANOVA procedures to estimate the magnitude of multiple sources of measurement errors. For example, in a writing test, G-theory allows one to separate observed test score variance into multiple facets, so that one can determine the relative contributions of various sources such as items, raters, or occasions (Brennan, 2001a; Shavelson \& Webb, 1981). This is particularly important given the complex nature of the assessments used in educational and psychological settings. With the information produced by G-theory, a

Zhehan Jiang

jiangzhehan@gmail.com

1 Pediatrics, Baylor College of Medicine, Houston, TX, USA

2 Assessment Design and Delivery, National Conference of Bar Examiners, Madison, WI, USA

3 Psychology, University of South Carolina, Columbia, SC, USA

4 Educational Studies, University of South Carolina, Columbia, SC, USA researcher can understand the quality of an assessment and make decisions accordingly (e.g., increasing the number of items or decreasing the number of raters). Multivariate Gtheory (mG-theory), as its name suggests, expands the univariate conception of measurement procedures into multivariate scenarios (Joe \& Woodward, 1976; Marcoulides, 1995; Woodward \& Joe, 1973), which tend to be more practically useful in many research contexts. For example, mG-theory allows researchers to study measurement designs for which more than a single dimension or subtest is of interest. Such analyses incorporate covariance components and/or correlations across multiple dimensions into a single overall analysis.

The information provided by $\mathrm{mG}$-theory can be useful when studying complex measurement designs to help determine when to combine two or more scores into a single score, to identify halo errors (Lance, LaPointe, \& Stewart, 1994) and other types of correlated errors, and to evaluate the consistency of different sources of errors across the multiple test scores of interest. Furthermore, ignoring the correlations of the dimensions could be detrimental, because it typically results in biased estimates (Rao \& Mudholkar, 1967). Applications of mG-theory can be found in various areas. For instance, $\mathrm{Nu}$ ßbaum (1984) adopted mG-theory to study students' watercolor painting skills in multiple dimensions, while $\mathrm{Wu}$ and Tzou (2015) analyzed standard setting data over multiple content areas and levels of proficiency. Clauser, Harik, and Margolis (2006) studied multiple sources of errors on a performance test where physicians interacted with several 
standardized patients and were evaluated on multiple skill domains (e.g., physical exam and communication), and Raymond and Jiang used mG-theory to evaluate the quality of subscores on multiple-choice tests (Jiang \& Raymond, 2018; Raymond \& Jiang, 2019). Brennan (2001a) provides more comprehensive examples of measurement problems that require $\mathrm{mG}$-theory for scientific decision-making procedures.

\section{Software for estimation}

Estimates of variance-covariance components for randomand fixed-effect measurement designs are required by $\mathrm{mG}$ theory. The variance-covariance components provide the foundation for consistency of the measurement(s), where in a testing scenario, reliability is one of form of such consistency. One of the simplest designs, using writing test score as an example, is where examinees respond to test items, and test items are nested within multiple content categories (i.e., dimensions). Examinees and items are random effects, while the content categories are fixed. Brennan (2001a) refers to this as the table of specifications design. However, the designs quickly become more complex as additional factors are added (e.g., raters and occasions). At present, mG-theory relies primarily on two software programs for estimating variance-covariance components: mGENOVA (Brennan, 2001b) and the BUGS/ JAGS suite of programs. As a frequentist product, mGENOVA is an ANSI C program powered by mean square error calculations, which are typically seen in traditional repeated-measures ANOVA estimations. These ANOVA approaches can also be realized in a structural equation modeling framework (Jiang, Walker, Shi, \& Cao, 2018; Marcoulides, 1996; Raykov \& Marcoulides, 2006). On the other hand, BUGS/JAGS refers to three variants sharing highly similar algorithmic functionalities: WinBUGS (Lunn, Thomas, Best, \& Spiegelhalter 2000; Spiegelhalter, Thomas, Best, \& Lunn 2003), OpenBUGS (Thomas, O'Hara, Ligges, \& Sturtz 2006), and JAGS (Plummer 2003, 2010). These programs manage the estimation from a Bayesian perspective, which enables features such as the incorporation of prior distributions, missing data imputation, and posterior distributions for variance/ covariance estimates (LoPilato, Carter, \& Wang, 2015).

Because of the algorithmic differences, mGENOVA is computationally faster than BUGS/JAGS, and can process a large number of observations (Brennan, 2001b). However, mGENOVA is limited with respect to handling unbalanced designs and sparse or missing data, and produces negative variance estimates under certain conditions. In contrast, BUGS/JAGS uses a Monte Carlo simulation paradigmrandom sampling strategy - that almost always consumes more processing time than frequentist approaches, for which the updating steps and directions are deterministic (Jiang \& Skorupski, 2018).
Despite the fact that the two software programs process data in different ways, they do share a common feature: both are independent, stand-alone software programs that are not compatible with R software (R Development Core Team, 2019). The $R$ environment has become the preferred choice in statistical analysis across numerous disciplines (Chen, Dios, Mili, Wu, \& Wang, 2005). The prevalence of R software in behavioral and educational research has led to the creation of many packages/functions for specific inquires (Li \& Baron, 2011, p. 5). For example, the Rethomics package (Geissmann, Rodriguez, Beckwith, \& Gilestro, 2019) provides a framework for analyzing high-throughput behavioral data, and the cat $R$ package (Magis \& Barrada, 2017) is designed to manage computer-adaptive testing. The present paper describes and illustrates how the glmmTMB package (Brooks et al., 2017) can be adapted to estimate the variance-covariance components required for mG-theory. The primary advantage of this approach is that processing and analysis of data can all be performed in the R computing environment ( $\mathrm{R}$ Development Core Team, 2019).

\section{Linear mixed-effect model and multivariate generalizability theory}

Moore (2016) adopted the lme4 package (Bates, Mächler, Bolker, \& Walker, 2015) to create the gtheory package to handle G-theory estimation in a linear mixed-effect model (LMM) framework. Jiang and Skorupski (2018) extended the lme4 approach (Bates et al., 2015) to additional G-theory designs; similar works can be found in Huebner and Lucht (2019). This paper follows Jiang and Skorupski's approach (2018) and demonstrates procedures for gearing LMM analysis towards $\mathrm{mG}$-theory estimation. Prior to introducing the R steps, the concepts of LMM are reviewed.

Linear mixed-effect models, also known as hierarchical linear models or multilevel models, comprise a class of statistical models specified for analyzing complex designs with clustering or nested structures (Raudenbush \& Bryk, 2002). To illustrate, if multiple high schools with multiple students are recruited into a research program, the students from the same high schools will have more in common with each other than with those from different high schools. Because students are nested within high schools, an LMM should be considered. As a special form, a cross-classified LMM is used when non-exclusive groups/structures exist: students from the same high school do not necessarily came from the same middle school, and vice versa. Therefore, the relation between the high schools and the middle schools is called a crossclassification (Goodman, 1985). For each nesting structure (e.g., high schools), variance can be estimated in the LMM to determine the significance of the difference among the entities. That said, if the between-school variance is not 
statistically significant, the hypothesis of no between-school difference cannot be rejected. In this instance, the higher-level entities, or schools, are treated as "random effects." In an educational testing setting, if examinees take all items, which are further scored by all raters, the cross-classified structure describes the relation between the item (random) effect and the rater (random) effect.

Mathematically, a typical two-level LMM with three predictors can be found in Eq. 1: $Y$ is the dependent variable; $\beta_{0}$ is the intercept; $\beta_{1}, \beta_{2}$, and $\beta_{3}$ are the slopes of predictors $X_{1}$, $X_{2}$, and $X_{3} ; \gamma_{00}, \gamma_{10}, \gamma_{20}$, and $\gamma_{30}$ are the means of $\beta_{0}, \beta_{1}, \beta_{2}$, and $\beta_{3}$, respectively, while $u_{0}, u_{1}, u_{2}$, and $u_{3}$ are the level-2 residuals; and finally, $r$ represents the level-1 residual:

$$
\begin{aligned}
Y=\beta_{0}+ & \beta_{1} X_{1}+\beta_{2} X_{2}+\beta_{3} X_{3}+r, \\
& \beta_{0}=\gamma_{00}+u_{0}, \\
& \beta_{1}=\gamma_{10}+u_{1}, \\
\beta_{2} & =\gamma_{20}+u_{2}, \\
\beta_{3} & =\gamma_{30}+u_{3} .
\end{aligned}
$$

Conventionally, $r$ is assumed to follow a univariate normal distribution $r \sim \mathrm{N}(0, \epsilon)$. On the other hand, $u_{0}, u_{1}, u_{2}$, and $u_{3}$ can be framed with more complex structures. Let $\boldsymbol{u}=\left[u_{0}, u_{1}, u_{2}, u_{3}\right]^{T} ; \boldsymbol{u}$ is assumed to follow a multivariate normal distribution $\boldsymbol{u} \sim \mathrm{MN}(\mathbf{0}$, $\Sigma$ ), where $\Sigma$ is a $4 \times 4$ matrix whose shape can be customized accordingly. Figure 1 shows typical specifications of $\Sigma$ : panels 1 to 4 are diagonal, unstructured, compound symmetric, and firstorder autoregressive covariance matrices, respectively. These structures are adopted according to theoretical assumptions or operational guidelines, while the results of fitting different structures can also be compared from an empirical prospective.

To illustrate how mG-theory can be converted to an LMM, two typical mG-theory designs are introduced: $p \bullet \times i^{0}$ and $p \bullet \times$ $i \bullet$. The notations and symbols follow Brennan's delineation (2001a) for labeling of random effects: $p$ is the person effect and $i$ is the item effect, where $p \bullet \times i^{0}$ indicates that different sets of items are nested within each dimension, and $p \bullet x \cdot \bullet$ indicates that items nested within all dimensions are identical. Examples in Jiang and Skorupski (2018) are reproduced to assist in explaining. As seen in Fig. 2, 200 examinees take a test that consists of 30 items in total. Items $i 1$ to $i 10$ are nested within the first dimension $v 1$, items $i 11$ to $i 20$ belong to the second dimension $v 2$, and the rest of the items measure the last

$$
\begin{aligned}
& \Sigma=\left[\begin{array}{cccc}
\sigma_{1}^{2} & 0 & 0 & 0 \\
0 & \sigma_{2}^{2} & 0 & 0 \\
0 & 0 & \sigma_{3}^{2} & 0 \\
0 & 0 & 0 & \sigma_{4}^{2}
\end{array}\right] \\
& 2=\left[\begin{array}{cccc}
\sigma_{1}^{2} & \sigma_{12} & \sigma_{13} & \sigma_{14} \\
\sigma_{12} & \sigma_{2}^{2} & \sigma_{23} & \sigma_{24} \\
\sigma_{13} & \sigma_{23} & \sigma_{3}^{2} & \sigma_{34} \\
\sigma_{14} & \sigma_{24} & \sigma_{34} & \sigma_{4}^{2}
\end{array}\right] \\
& \Sigma=\left[\begin{array}{llll}
1 & p & p & p \\
p & 1 & p & p \\
p & p & 1 & p \\
p & p & p & 1
\end{array}\right] \\
& \Sigma=\left[\begin{array}{cccc}
1 & p & p^{2} & p^{3} \\
p & 1 & p & p^{2} \\
p^{2} & p & 1 & p \\
p^{3} & p^{2} & p & 1
\end{array}\right]
\end{aligned}
$$

Fig. 1 Covariance structure examples dimension $v 3$. Each examinee responds to each test item only once. The design can be expressed as:

$$
\left(\begin{array}{l}
X_{1 p i} \\
X_{2 p i} \\
X_{3 p i}
\end{array}\right)=\left(\begin{array}{l}
\mu_{1} \\
\mu_{2} \\
\mu_{3}
\end{array}\right)+\left(\begin{array}{c}
\theta_{1 p} \\
\theta_{2 p} \\
\theta_{3 p}
\end{array}\right)+\left(\begin{array}{c}
\theta_{1 i} \\
\theta_{2 i} \\
\theta_{3 i}
\end{array}\right)+\left(\begin{array}{c}
\epsilon_{1} \\
\epsilon_{2} \\
\epsilon_{3}
\end{array}\right) .
$$

$X$ represents the actual responses, and the subscripts 1 to 3 indicate dimension $v$, where $p$ and $i$ define the examinee and the item, respectively. Equation 2 shows that a response for an item $i$ on dimension $v$ can be broken down into the grand mean $\mu$, the examinee's contribution $\theta_{p}$, the item effect $\theta_{i}$, and the error term $\epsilon$. It is assumed that all $\boldsymbol{\theta}$ and $\boldsymbol{\epsilon}$ follow multivariate normal distributions whose mean vectors are $\mathbf{0}$ in $\mathrm{mG}$-theory. Correspondingly, the variance-covariance matrices from Eq. 2 can be written as three blocks:

$$
\begin{aligned}
{\left[\begin{array}{ccc}
\sigma_{1 X_{p i}}^{2} & \sigma_{12 X_{p i}}^{2} & \sigma_{13 X_{p i}}^{2} \\
\sigma_{12 X_{p i}}^{2} & \sigma_{2 X_{p i}}^{2} & \sigma_{23 X_{p i}}^{2} \\
\sigma_{13 X_{p i}}^{2} & \sigma_{23 X_{p i}}^{2} & \sigma_{3 X_{p i}}^{2}
\end{array}\right] } & =\left[\begin{array}{ccc}
\sigma_{1 p}^{2} & \sigma_{1 p 2 p}^{2} & \sigma_{1 p 3 p}^{2} \\
\sigma_{1 p 2 p}^{2} & \sigma_{2 p}^{2} & \sigma_{2 p 3 p}^{2} \\
\sigma_{1 p 3 p}^{2} & \sigma_{2 p 3 p}^{2} & \sigma_{3 p}^{2}
\end{array}\right] \\
& +\left[\begin{array}{ccc}
\sigma_{1 i}^{2} & 0 & 0 \\
0 & \sigma_{2 i}^{2} & 0 \\
0 & 0 & \sigma_{3 i}^{2}
\end{array}\right]+\left[\begin{array}{ccc}
\sigma_{1 e}^{2} & 0 & 0 \\
0 & \sigma_{2 e}^{2} & 0 \\
0 & 0 & \sigma_{3 e}^{2}
\end{array}\right] .
\end{aligned}
$$

Now consider the $p \bullet \times i \bullet$ design, which also follows Eq. 2 but with different variance-covariance structures. Figure 3 shows a typical example of the design: 200 examinees take a 10 -item test. These items measure three dimensions and therefore are scored three times. This situation would arise, for example, in a writing test where students' essay responses are graded on grammar, style, and content. The variancecovariance structure can be formulated as:

$$
\begin{gathered}
{\left[\begin{array}{ccc}
\sigma_{1 X_{p i}}^{2} & \sigma_{12 X_{p i}}^{2} & \sigma_{13 X_{p i}}^{2} \\
\sigma_{12 X_{p i}}^{2} & \sigma_{2 X_{p i}}^{2} & \sigma_{23 X_{p i}}^{2} \\
\sigma_{13 X_{p i}}^{2} & \sigma_{23 X_{p i}}^{2} & \sigma_{3 X_{p i}}^{2}
\end{array}\right]=\left[\begin{array}{ccc}
\sigma_{1 p}^{2} & \sigma_{1 p 2 p}^{2} & \sigma_{1 p 3 p}^{2} \\
\sigma_{1 p 2 p}^{2} & \sigma_{2 p}^{2} & \sigma_{2 p 3 p}^{2} \\
\sigma_{1 p 3 p}^{2} & \sigma_{2 p 3 p}^{2} & \sigma_{3 p}^{2}
\end{array}\right]} \\
+\left[\begin{array}{ccc}
\sigma_{1 i}^{2} & \sigma_{12 i}^{2} & \sigma_{1 i 3 i}^{2} \\
\sigma_{1 i 2 i}^{2} & \sigma_{2 i}^{2} & \sigma_{2 i 3 i}^{2} \\
\sigma_{13 i i}^{2} & \sigma_{2 i 3 i}^{2} & \sigma_{3 i}^{2}
\end{array}\right]+\left[\begin{array}{ccc}
\sigma_{1 e}^{2} & \sigma_{1 e 2 e}^{2} & \sigma_{1 e 3 e}^{2} \\
\sigma_{1 e 2 e}^{2} & \sigma_{2 e}^{2} & \sigma_{2 e e 3}^{2} \\
\sigma_{1 e 3 e}^{2} & \sigma_{2 e 3 e}^{2} & \sigma_{3 e}^{2}
\end{array}\right]
\end{gathered}
$$

One can see that the examinee effect in the $p \bullet \times i^{0}$ design is distributed in the unstructured shape, while others follow the diagonal structure. In contrast, the $p^{\bullet} \times i \bullet$ design adopts the unstructured structure for all variance and covariance components. These structures are specified according to both theory and actual design. For instance, in the $p^{\bullet} \times i^{0}$ design, the given items nested in each dimension come from different item sets, and thus the variance-covariance matrix should be of a diagonal structure instead of unstructured. On the other hand, since examinees nested in the dimensions are from the same group of respondents, the covariance components (i.e., the correlations) cannot be ignored. 


\begin{tabular}{|c|c|c|c|c|c|c|c|c|c|c|c|c|}
\hline \multirow[b]{2}{*}{$p$} & \multicolumn{4}{|c|}{$\mathrm{v} 1$} & \multicolumn{4}{|c|}{$\mathrm{v} 2$} & \multicolumn{4}{|c|}{ v3 } \\
\hline & i1 & $i 2$ & $\ldots$ & $\mathrm{i} 10$ & i11 & $\overline{\mathrm{i} 2}$ & $\ldots$ & $i 20$ & $i 21$ & $\mathrm{i} 22$ & $\ldots$ & $\mathrm{i} 30$ \\
\hline 1 & $\mathrm{x}$ & $x$ & $\ldots$ & $\mathrm{x}$ & $\mathrm{x}$ & $\bar{x}$ & & $\mathrm{x}$ & $x$ & $\mathrm{x}$ & & $x$ \\
\hline 2 & $x$ & $x$ & $\ldots$ & $x$ & $x$ & $x$ & $\ldots$ & $x$ & $x$ & $x$ & $\ldots$ & $x$ \\
\hline$\ldots$ & $\ldots$ & $\ldots$ & $\ldots$ & $\ldots$ & $\ldots$ & $\ldots$ & $\ldots$ & $\ldots$ & $\ldots$ & $\ldots$ & $\ldots$ & $\ldots$ \\
\hline 200 & $x$ & $x$ & $\ldots$ & $\mathrm{x}$ & $x$ & $x$ & $\ldots$ & $x$ & $x$ & $x$ & $\ldots$ & $x$ \\
\hline
\end{tabular}

Fig. 2 A typical example of $p \bullet \times i^{0}$ design

One can rewrite $\mu_{1}=\mu+\Delta \mu_{1}, \mu_{2}=\mu+\Delta \mu_{2}$, and $\mu_{3}=\mu+$ $\Delta \mu_{3}$ from Eq. 2. Therefore, referring Eq. 2 to Eq. 1, $\mu$ can be regarded as $\beta_{0}$ (i.e., $\gamma_{00}$ ) and $u_{0}$ can be removed. At first glance, it appears that no predictors are used in the aforementioned $\mathrm{mG}$ theory designs. However, dummy variables can serve as predictors to indicate the dimension of a response. That said, a response labeled with $\left[\mathrm{X}_{1}, \mathrm{X}_{2}, \mathrm{X}_{3}\right]=[1,0,0]$ origins from dimension $v 1$, and $[0,1,0]$ and $[0,0,1]$ from dimensions $v 2$ and $v 3$, respectively. As seen above, if $\mu_{1}=\beta_{0}+\Delta \mu_{1}, \Delta \mu_{1}$ can be treated as $\gamma_{10}$ for a response in dimension $v 1$. The same idea applies to dimensions $v 2$ and $v 3$. Now, what remains in level 2 of Eq. 1 is $\boldsymbol{u}$. Unsurprisingly, since $\boldsymbol{u} \sim \mathrm{MN}(\mathbf{0}, \Sigma)$, all $\mathrm{mG}$-theory variance and covariance components can be wired to $\boldsymbol{u}$. Recall the examples illustrated in Figs. 2 and 3: responses are nested within examinees and items. Taking the examinee block, for example, $\left[u_{1}\right.$, $\left.u_{2}, u_{3}\right]^{T}$ is distributed with $\left[\begin{array}{ccc}\sigma_{1 p}^{2} & \sigma_{1 p 2 p}^{2} & \sigma_{1 p 3 p}^{2} \\ \sigma_{1 p 2 p}^{2} & \sigma_{2 p}^{2} & \sigma_{2 p 3 p}^{2} \\ \sigma_{1 p 3 p}^{2} & \sigma_{2 p 3 p}^{2} & \sigma_{3 p}^{2}\end{array}\right]$, where the number of the level-2 clusters is $P$ (i.e., the total number of the examinees). Given that a cross-classified relation can be built in the LMM, other blocks can then be formulated with the same flow. Therefore, $\left[u_{1}, u_{2}, u_{3}\right]^{T}$ can be further extended to $\left[u_{1 p}, u_{2 p}\right.$, $\left.u_{3 p}\right]^{T},\left[u_{1 i}, u_{2 i}, u_{3 i}\right]^{T}$, and $\left[u_{1 e}, u_{2 e}, u_{3 e}\right]^{T}$ to synthesize all three variance and covariance blocks in Eqs. 3 and 4. Last but not least, since mG-theory does not assume a univariate normal distribution, the $r$ term in the LMM needs to be fixed to 0 . This step is critical, as $\left[u_{1 e}, u_{2 e}, u_{3 e}\right]^{T}$ should already include the residual components; specifying the $r$ term can result in a redundant residual specification, which violates the assumption of unique residuals for each dimension in $\mathrm{mG}$-theory (Webb, Shavelson, \& Haertel, 2006). To be clear, if the $r$ term in the LMM were permissible, the final estimates would be "parameters in Eq. 4 plus a new $r$." At this point, the connection between LMMs and $\mathrm{mG}$-theory should be perceptible.

\section{The glmmTMB package}

The glmmTMB package (Brooks et al., 2017) offers comprehensive solutions to fit generalized linear mixed models and their extensions. Similar to the famous lme4 package (Bates et al., 2015), the glmmTMB package (Brooks et al., 2017) allows users to create a model with (1) different response types such as Gaussian, Poisson, and beta distributions; (2) link functions such as log, logit, identity, and complementary log-log; and (3) single or multiple random effects and fixed effects, and others. Jiang (2018) claimed that, due to the prohibition of correlated residuals, the lme 4 approach (and therefore the gtheory package) could not be extended to $\mathrm{mG}$-theory designs. To be clear, the lme 4 package (Bates et al., 2015) does not provide the capacity to specify different covariance structures for random effects: if a design contains two dimensions, all covariance structures are identical except the diagonal one; however, if the number of dimensions is larger than two, an unstructured covariance, which the lme 4 package (Bates et al., 2015) cannot afford, is needed to model the unique correlations between each pair of dimensions. In addition, for crossed designs, such as $p^{\bullet \times} i^{\bullet}$, the ability to obtain correlations for the residual components is required, and the lme4 package (Bates et al., 2015) is not capable of providing such a feature. The glmmTMB package (Brooks et al., 2017) possesses higher flexibility than the lme4 package (Bates et al., 2015) for specifying variance and covariance structures. In accordance with the connection between LMMs and mG-theory, the challenging estimation becomes achievable in $\mathrm{R}$ software.

\begin{tabular}{|c|c|c|c|c|c|c|c|c|c|c|c|c|}
\hline \multirow[b]{2}{*}{$\mathrm{p}$} & \multicolumn{4}{|c|}{ v1 } & \multicolumn{4}{|c|}{$\mathrm{v} 2$} & \multicolumn{4}{|c|}{ v3 } \\
\hline & i1 & $\mathrm{i} 2$ & $\ldots$ & $\mathrm{i} 10$ & i1 & $\mathrm{i} 2$ & $\ldots$ & $\mathrm{i} 10$ & i1 & $\mathrm{i} 2$ & $\ldots$ & $\mathrm{i} 10$ \\
\hline 1 & $\mathrm{x}$ & $\mathrm{x}$ & $\ldots$ & $\mathrm{x}$ & $\mathrm{x}$ & $x$ & & $\mathrm{x}$ & $x$ & $\mathrm{x}$ & & $\mathrm{x}$ \\
\hline 2 & $x$ & $x$ & $\ldots$ & $x$ & $x$ & $x$ & $\ldots$ & $x$ & $x$ & $x$ & $\ldots$ & $x$ \\
\hline$\ldots$ & $\ldots$ & $\ldots$ & $\ldots$ & $\ldots$ & $\ldots$ & $\ldots$ & $\ldots$ & $\ldots$ & $\ldots$ & $\ldots$ & $\ldots$ & $\ldots$ \\
\hline 200 & $\mathrm{x}$ & $x$ & $\ldots$ & $x$ & $\mathrm{x}$ & $x$ & $\ldots$ & $x$ & $x$ & $x$ & $\ldots$ & $\mathrm{x}$ \\
\hline
\end{tabular}

Fig. 3 A typical example of $\mathrm{p} \bullet \times \mathrm{i} \bullet$ design 
To increase speed and flexibility, the $\operatorname{glmm} T M B$ estimation is achieved using Template Model Builder, and its interface is designed to be similar to the lme4 package (Bates et al., 2015) ; therefore, the lme 4 means of specifying models and marking notations is inherent in the glmmTMB package (Brooks et al., 2017). Readers interested in seeing detailed syntax are referred to Bates (2005) and to Doran, Bates, Bliese, and Dowling (2007).

Prior to the analysis, datasets should be converted to a long format. As Table 1 illustrates, in a long format, each row of the dataset should be one response, where the columns contain the identity. For example, in the fourth row (i.e., ID $=4$ ), the response, 46 , is from examinee \#4 in item \#1 and belongs to the first dimension. Therefore, $d 1$ is 1 , whereas $d 2$ and $d 3$ are 0 , as they are the dummy coding variables for the dimension. Note that in a long format, statistically one will not encounter missingness, as NA (i.e., not available) responses need not be present in the dataset. The process of reshaping a wide format into a long format can be found in Lander (2014, p. 149). The example demonstrated in Fig. 2 can be modeled via the glmmTMB package (Brooks et al., 2017) as shown below.

mGtheory<-glmmTMB(

Response $\sim \mathrm{d} 1+\mathrm{d} 2+\mathrm{d} 3+\mathrm{us}(0+\mathrm{d} 1+\mathrm{d} 2+\mathrm{d} 3 \mid$ Examinee $)+\operatorname{diag}(0+\mathrm{d} 1+\mathrm{d} 2+\mathrm{d} 3 \mid$ Item $)+$ $\operatorname{diag}(0+\mathrm{d} 1+\mathrm{d} 2+\mathrm{d} 3 \mid \mathrm{ID})$

data $=$ dataset,

dispformula $=\sim 0$,

control $=$ glmmTMBControl $($ optCtrl=list(iter.max=1e3,eval.max=1e3)

)

The names of the variables used in the $\mathrm{R}$ syntax follow the convention shown in Table 1 . The notation " " bridges the linear relationship between "Response" and the dummy-coded "Dimension." By default, the $\operatorname{glmm} T M B$ package (Brooks et al., 2017) generates an intercept, which is $\beta_{0}$ in an LMM context. The coefficients of "Dimension"- "d1" to "d3"- are the estimates of $\Delta \mu_{1}$ to $\Delta \mu_{3}$, respectively. As addressed above, the example in Fig. 2 contains three random-effect blocks. The "us" and "diag" correspond to the unstructured and the diagonal structures, respectively. One can see that this specification aligns with Eqs. 1 and 2. Note that " 0 " should be included in all blocks to ensure that the intercept component is not automatically included in the blocks' formulas. The "data" navigates the name of the dataset, and the
Table 1 The dataset used in the analysis in a long format

\begin{tabular}{cccccccc}
\hline ID & Examinee & Item & Dimension & $\mathrm{d} 1$ & $\mathrm{~d} 2$ & $\mathrm{~d} 3$ & Response \\
\hline 1 & 1 & 1 & 1 & 1 & 0 & 0 & 47 \\
2 & 2 & 1 & 1 & 1 & 0 & 0 & 42 \\
3 & 3 & 1 & 1 & 1 & 0 & 0 & 42 \\
4 & 4 & 1 & 1 & 1 & 0 & 0 & 46 \\
$\ldots$ & $\ldots$ & $\ldots$ & $\ldots$ & $\ldots$ & $\ldots$ & $\ldots$ & $\ldots$ \\
3001 & 1 & 11 & 2 & 0 & 1 & 0 & 52 \\
3002 & 2 & 11 & 2 & 0 & 1 & 0 & 53 \\
$\ldots$ & $\ldots$ & $\ldots$ & $\ldots$ & $\ldots$ & $\ldots$ & $\ldots$ & $\ldots$ \\
5998 & 198 & 30 & 3 & 0 & 0 & 1 & 61 \\
5999 & 199 & 30 & 3 & 0 & 0 & 1 & 63 \\
6000 & 200 & 30 & 3 & 0 & 0 & 1 & 64 \\
\hline
\end{tabular}

"dispformula" defines the shape of the residuals. To reiterate, the $r$ term in LMMs will be fixed to 0 to estimate mG-theory. Therefore, " $=\sim 0$ " is entered to force the constraint. Finally, the "control" is simply a batch of commands for algorithmic configurations, such as the number of iterations and other stopping criteria. Alternatively, if the attribute of the dimension variable in the dataset (i.e., "Dimension" in Table 1) is converted to factor, the modeling specification part in the $\operatorname{glmm} T M B$ syntax can be rewritten as: Response $\sim$ Dimension+us $(0+$ Dimension |Examinee $)+\operatorname{diag}(0+$ Dimension $\mid$ Item $)+\operatorname{diag}(0+$ Dimension (ID). To adjust the $\operatorname{glmm} T M B$ syntax to $p \bullet \times$ $i \bullet$ design, the "diag" should be replaced with the "us" to update variance structures appropriately.

\section{Data analysis example}

In this section, the $\operatorname{glmm} T M B \mathrm{R}$ code for the $p \bullet \times i^{0}$ design is used to analyze a simulated dataset. This is conducted for the purpose of providing a tutorial, not for performing a largescale simulation study with numerous replications. That said, parameter recovery is not part of the present study. The dataset generation and true value specifications follow the practice by Jiang and Skorupski (2018). That is, a one-facet fully crossed design is employed where the number of dimensions is three. Mathematically, the design can be expressed as Eq. 2. The true values for $\boldsymbol{\mu}$ are $[3,4,5]$, where the variance components are specified as below in Eq. 5:

$$
\begin{aligned}
{\left[\begin{array}{ccc}
\sigma_{1 X_{p i}}^{2} & \sigma_{12 X_{p i}}^{2} & \sigma_{13 X_{p i}}^{2} \\
\sigma_{12 X_{p i}}^{2} & \sigma_{2 X_{p i}}^{2} & \sigma_{23 X_{p i}}^{2} \\
\sigma_{13 X_{p i}}^{2} & \sigma_{23 X_{p i}}^{2} & \sigma_{3 X_{p i}}^{2}
\end{array}\right]=\left[\begin{array}{ccc}
4 & 3 & 2 \\
3 & 5 & 1 \\
2 & 1 & 4.5
\end{array}\right] } \\
+\left[\begin{array}{ccc}
6.5 & 0 & 0 \\
0 & 8.5 & 0 \\
0 & 0 & 4.5
\end{array}\right]+\left[\begin{array}{ccc}
4.1 & 0 & 0 \\
0 & 5.1 & 0 \\
0 & 0 & 2.3
\end{array}\right] .
\end{aligned}
$$


After the variance and covariance components are estimated, the generalizability coefficient, known as the G-coefficient, in mG-theory can also be calculated. The Gcoefficient in mG-theory is defined as:

$\rho_{\delta}^{2}=\frac{\boldsymbol{a}^{\prime} \sum_{p} \boldsymbol{a}}{\boldsymbol{a}^{\prime} \sum_{p} \boldsymbol{a}+\boldsymbol{a}^{\prime} \sum_{\delta} \boldsymbol{a} / \boldsymbol{n}_{\boldsymbol{i}}}$.

$\sum_{p}$ is the examinee effect covariance matrix, while $\sum_{\delta}$ is the error covariance matrix, and $n_{i}$ is the number of items. The $\boldsymbol{a}$ vector is a weighting scheme defining the relative importance of each dimension (see Wang \& Stanley, 1970 for an overview). Although there are different ways to specify $\boldsymbol{a}$ (Brennan, 2001a; Marcoulides, 1994; Srinivasan \& Shocker, 1973), to simplify the illustration, an equal weight scheme is adopted and therefore results in the true $\rho_{\delta}^{2}=0.98$. In addition to adopting equal weight scheme or other nominal specifications, effective weight and data-driven weight schemes are developed to offer different perspectives (see Appendix for the calculation). Specifically, nominal weights express the researcher's judgment regarding the relative importance of the various levels of a facet, where effective weights indicate the relative statistical contributions, and data-driven weighting schemes use iterative approaches to optimize generalizability (Marcoulides, 1994). Weight schemes have a great impact on decision-making in evaluating measurement instruments. Therefore, defining weights should be supported with evidence and thorough literature reviews. In the present scheme, the number of persons and the number of items is 200 and 30, respectively, while
Table 3. The estimates yielded by the $\operatorname{glmm} T M B$ package

Random-effects (co)variances:

Conditional model:

\begin{tabular}{|c|c|c|c|}
\hline Groups & Name & Std. Dev. & Corr \\
\hline \multirow[t]{3}{*}{ Examinee } & $\mathrm{d} 1$ & 1.8204895 & \\
\hline & $\mathrm{d} 2$ & 2.0899889 & 0.83 \\
\hline & $\mathrm{d} 3$ & 1.9196811 & 0.580 .42 \\
\hline \multirow[t]{3}{*}{ Item } & $\mathrm{d} 1$ & 2.8143787 & \\
\hline & $\mathrm{d} 2$ & 2.72475390 .00 & \\
\hline & $\mathrm{d} 3$ & 1.4266613 & 0.000 .00 \\
\hline \multirow[t]{3}{*}{ ID } & $\mathrm{d} 1$ & 2.0428350 & \\
\hline & $\mathrm{d} 2$ & 2.2564543 & 0.00 \\
\hline & $\mathrm{d} 3$ & 1.5667994 & 0.000 .00 \\
\hline Residual & 0.0001221 & & \\
\hline \multicolumn{4}{|c|}{$\begin{array}{l}\text { Number of obs: } 6000 \text { / Conditional model: Examinee, 200; Item, 30; II } \\
6000\end{array}$} \\
\hline \multicolumn{4}{|c|}{ Fixed Effects: } \\
\hline \multicolumn{4}{|c|}{ Conditional model: } \\
\hline (Intercept) & $\mathrm{d} 1$ & $\mathrm{~d} 2$ & $\mathrm{~d} 3$ \\
\hline 44.45 & 5.75 & 14.85 & 25.85 \\
\hline
\end{tabular}

each dimension comprises 10 items. The preview of the dataset in a long format is listed in Table 1; in total, there are 6000 responses. The computer used is a Lenovo IdeaPad with $8 \mathrm{~GB}$ RAM and a $2.6 \mathrm{GHz}$ i7 6th Gen 4core Intel processor. To compare the estimations, the dataset was fed into mGENOVA (Brennan, 2001b), and the estimates of interest are presented in Table 2.

Table 2 The estimates yielded by mGENOVA

\begin{tabular}{|c|c|c|c|}
\hline \multicolumn{2}{|c|}{ INFORMATION ABOUT VARIABLE 1: } & \multicolumn{2}{|l|}{ First } \\
\hline \multicolumn{2}{|c|}{ Grand mean: } & \multicolumn{2}{|l|}{50.20154} \\
\hline \multicolumn{2}{|c|}{ INFORMATION ABOUT VARIABLE 2: } & \multicolumn{2}{|l|}{ Second } \\
\hline \multicolumn{2}{|c|}{ Grand mean: } & \multicolumn{2}{|l|}{59.30468} \\
\hline \multicolumn{2}{|c|}{ INFORMATION ABOUT VARIABLE 3: } & \multicolumn{2}{|l|}{ Third } \\
\hline \multicolumn{2}{|c|}{ Grand mean: } & \multicolumn{2}{|l|}{70.30240} \\
\hline \multicolumn{4}{|c|}{ ESTIMATED G STUDY VARIANCE AND COVARIANCE COMPONENTS } \\
\hline Note. & $\begin{array}{l}\text { Lower di } \\
\text { Upper di }\end{array}$ & Upper diagonal elements are correlations. & \\
\hline Effect & First & Second & Third \\
\hline \multirow[t]{3}{*}{$\mathrm{p}$} & 3.28212 & 0.83023 & 0.57758 \\
\hline & 3.17494 & 4.45575 & 0.40256 \\
\hline & 2.00298 & 1.62659 & 3.66417 \\
\hline$i$ & 8.76900 & 8.17218 & 2.25605 \\
\hline $\mathrm{pi}$ & 4.09697 & 5.01437 & 2.33697 \\
\hline
\end{tabular}


The results produced by the $\operatorname{glmm} T M B$ package (Brooks et al., 2017) are presented in Table 3. Note that the estimates are the standard deviations and the correlations, instead of the variance and the covariance. As one can see, the results returned by mGENOVA (Brennan, 2001b) and the glmmTMB package (Brooks et al., 2017) are similar, meaning that the proposed approach indeed works and can be substituted for mGENOVA (Brennan, 2001b) when one prefers to analyze data using R software. While they are not substantial, the discrepancies reflect the functional differences in the selected algorithms: the glmmTMB package (Brooks et al., 2017) is powered by automatic differentiation to estimate model gradients and the Laplace approximation for handling random effects, whereas mGENOVA (Brennan, 2001b) is engineered by an ANOVA-based algorithm targeting expected mean squares. It is unsurprising that different algorithms, or different settings within the same algorithm, can produce different results, given the same dataset. Similarly, packages such as the lme4 (Bates et al., 2015) and the $\operatorname{glmm} T M B$ are known to have a certain likelihood of producing inconsistent mixed-model estimates (Bolker, 2017).

Further, even though the dispersion was constrained to be zero, the residual estimate is not exactly zero in Table 3, as the algorithm is approximation-based. The grand means (i.e., the intercepts) can be calculated by $44.45+5.75,44.45+14.85$, and $44.45+25.85$ for the $\mu$ estimate. The $\widehat{\rho_{\delta}^{2}}$ can be obtained via Eq. 7 as 0.96 , which is slightly lower than the true value. The correlation terms are all zeros for the "Item" and the "ID" blocks in Table 3, as they were specified to follow the diagonal structure, in alignment with the present design. As a one-time simulation, these differences are expected, but they also warrant caution: since mG-theory is essentially an LMM variant, the random effects require a large number of observations and/or clusters to ensure that they are capable of both (1) representing the properties of the population and (2) recovering the model parameters (Stegmueller, 2013). In the data analysis example, however, the numbers of fixed-effect levels are not sufficiently large (e.g., 10 levels of the item facet per dimension), so both the generated dataset and its analysis tend to deviate from the true values.

\section{Discussion}

This paper presents a new approach for estimating the variance and covariance components of mG-theory. Compared with G-theory, mG-theory offers researchers perspectives concerning the relations between dimensions, which are critical to measurement instrument developers interested in studying subtests and their variance differences. Therefore, the estimates in each dimension indicate how much variance a certain facet can contribute to the corresponding dimension. For instance, in the analysis example, the item variance estimates for the first two dimensions are larger than that of the last dimension; this finding may encourage researchers to further investigate the sources of variance in the uneven distribution across dimensions.

With the flexibility of the $\operatorname{glmm} T M B$ package (Brooks et al., 2017), users can tune LMMs to mG-theory such that the analysis can all be conducted in $\mathrm{R}$ software. The proposed method was also proven to yield results similar to mGENOVA (Brennan, 2001b) and therefore can serve as an alternative. Given that the $\operatorname{glmmTMB}$ package (Brooks et al., 2017) requires the dataset to be in a long format, estimations can still be made despite missing values, although biases can occur if the missingness is attributable to two patterns: missing at random and missing not at random. On the other hand, mGENOVA (Brennan, 2001b) does not allow the dataset to contain missing cells.

Like any other method, the proposed approach has limitations. The computational speed of the glmmTMB package (Brooks et al., 2017) can be significantly lower than mGENOVA (Brennan, 2001b) when a large dataset is used. In the previous section, the $\operatorname{glmm} T M B$ package (Brooks et al., 2017) required more than five seconds to converge, while mGENOVA (Brennan, 2001b) produced results immediately. As mGENOVA (Brennan, 2001b) algorithms are not matrix-based (Brennan, 2001a, p. 388), matrix operations deployed in the glmmTMB package (Brooks et al., 2017) can be avoided in mGENOVA (Brennan, 2001b), so the computation tends to be faster with the latter software. In addition to computational speed, a lack of intrinsic G-theory features in the software makes the proposed approach less appealing if comprehensive $\mathrm{mG}$-theory results are preferred. For instance, sequential research addressing the practical application of a measurement procedure, called a decision (D) study, is incorporated in mGENOVA (Brennan, 2001b). A D study can help users design a measurement procedure that reduces errors at selected facets. To illustrate, a D study for writing test scores can inform users of the degree of impact on the G-coefficient by changing the number of raters within a budget limit. As non-mGtheoryspecific software, the glmmTMB package (Brooks et al., 2017) can achieve the same functionality only by constructing a separate model for the decisional references. Thus, further research directions could include constructing a comprehensive $\mathrm{mG}$-theory $\mathrm{R}$ package on top of the glmmTMB package (Brooks et al., 2017); for instance, users could deploy the package directly to produce a generalizability index for $\mathcal{G}$ score profiles or to specify different designs with a simple parameter change. 


\section{Appendix}

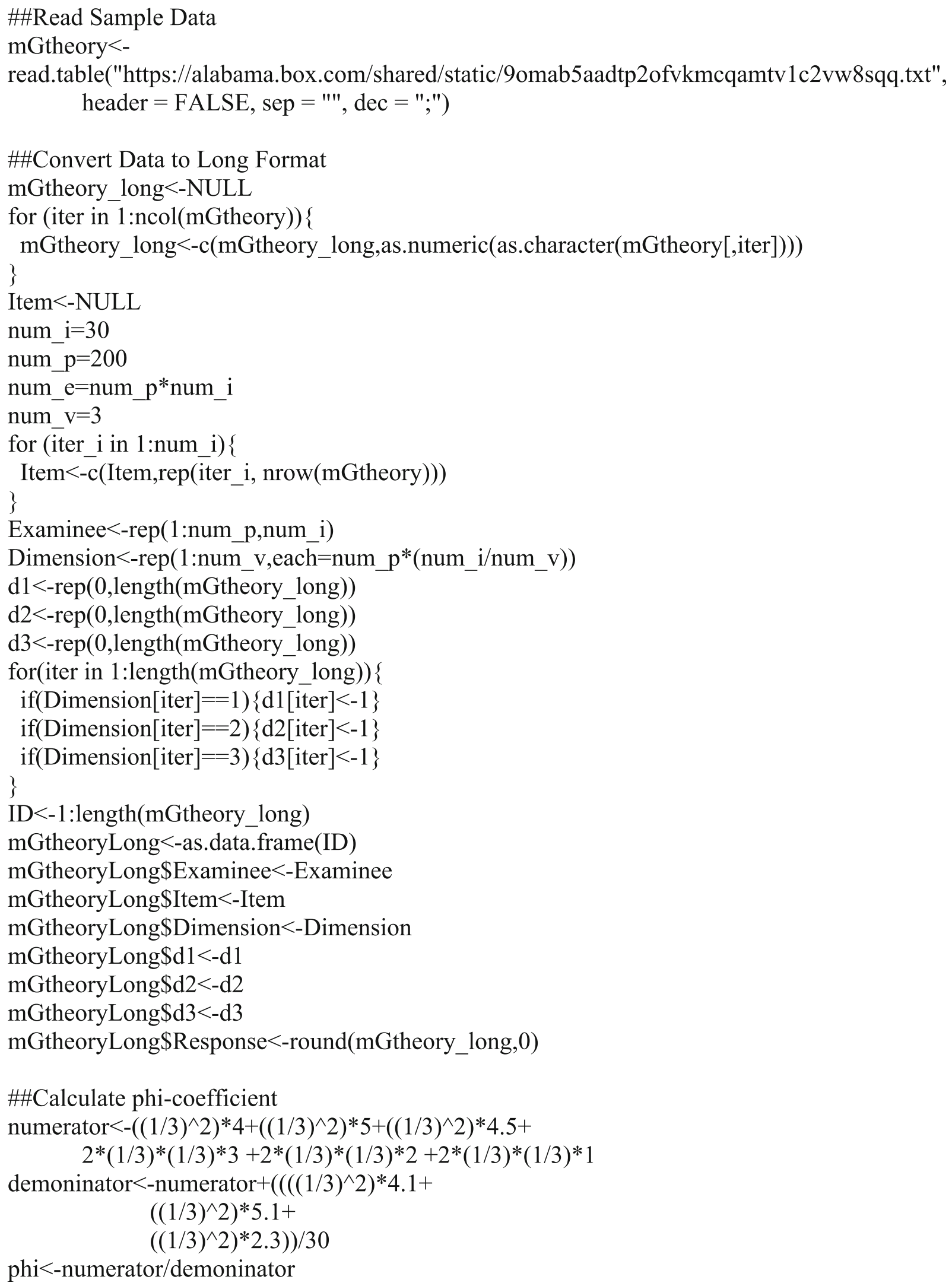


\#\#Use glmmTMB to estimate $\mathrm{p} \_$dot*i_circle mG-theory

library (glmmTMB)

glmmTMB $($ Response $\sim \mathrm{d} 1+\mathrm{d} 2+\mathrm{d} 3+\mathrm{us}(0+\mathrm{d} 1+\mathrm{d} 2+\mathrm{d} 3 \mid$ Examinee $)+\operatorname{diag}(0+\mathrm{d} 1+\mathrm{d} 2+\mathrm{d} 3 \mid$ Item $)+\operatorname{diag}(0+$ $\mathrm{d} 1+\mathrm{d} 2+\mathrm{d} 3 \mid \mathrm{ID})$, data $=\mathrm{mGtheoryLong,}$

dispformula $=\sim 0$,

control $=$ glmmTMBControl $($ optCtrl=list $($ iter.max $=1 \mathrm{e} 4$, eval.max=1e4) $))$

\#\#Alternative way to execute glmmTMB for $\mathrm{p}$ _dot*i_circle mG-theory

mGtheoryLong\$Dimension<-as.factor(mGtheoryLong\$Dimension)

glmmTMB(Response $\sim$ Dimension+us(Dimension|Examinee) + diag(Dimension|Item) + diag(Dime nsion| ID),

data $=$ mGtheoryLong,

dispformula $=\sim 0$,

control $=$ glmmTMBControl $($ optCtrl=list $($ iter $\cdot \max =1 \mathrm{e} 4$, eval.max=1e4))

\#\#Use glmmTMB to estimate $\mathrm{p} \_$dot*i_dot $\mathrm{mG}$-theory

glmmTMB(Response $\sim$ Dimension+us(Dimension|Examinee)+us(Dimension|Item) + us(Dimensio $\mathrm{n} \mid \mathrm{ID})$,

data $=$ mGtheoryLong,

dispformula $=\sim 0$,

control $=$ glmmTMBControl $($ optCtrl=list(iter.max $=1 \mathrm{e} 4$, eval. $\max =1 \mathrm{e} 4)))$

\#\#Use glmmTMB to estimate p_dot*i_dot*h_dot example in Jiang \& Skorupuski (2018)

mGtheory $2<-$

read.table("https://alabama.box.com/shared/static/fs02xjabzinwf85jt37wsp3rvbzpzrt7.txt", header = FALSE, sep = "", dec = ";")

mGtheory2_long<-NULL

for (iter in $1: \operatorname{ncol}(\mathrm{mGtheory} 2))\{$

mGtheory2_long<-c(mGtheory2_long,as.numeric(as.character(mGtheory2[,iter]))) \}

num_ $\mathrm{i}<-4$

num_ $\mathrm{h}<-3$

num_v $<-2$

num_p $<-100$

Item<-rep(rep(c(1:num_i),each=num_p),num_v*num_h)

Rater $<$-rep(rep $(c(1:$ num_h $)$, each $=$ num_p*num_i $)$,num_v)

Dimension<-rep(1:num_v,each=length(mGtheory2_long)/num_v)

Examinee $<-$ rep(c(1:num_p),length(mGtheory2_long)/num_p)

ID $<-1$ :length(mGtheory2_long)

mGtheoryLong2 $<$-as.data.frame(ID)

mGtheoryLong2\$Examinee $<$-Examinee

mGtheoryLong2\$Item $<$-Item

mGtheoryLong2\$Dimension $<$-as.factor(Dimension)

mGtheoryLong2\$Rater $<$-Rater

mGtheoryLong2 \$Response<-round(mGtheory2_long,0)

glmmTMB(Response $\sim$ Dimension+us(Dimension|Examinee)+us(Dimension|Item) + us(Dimensio $\mathrm{n} \mid$ Rater $)+$

us(Dimension|Examinee:Item)+us(Dimension|Item:Rater)+us(Dimension|Examinee:Rater)+ us(Dimension|ID),

data $=$ mGtheoryLong2,

dispformula $=\sim 0$,

control $=$ glmmTMBControl $($ optCtrl=list $($ iter.max $=1 \mathrm{e} 4$, eval.max $=1 \mathrm{e} 4)))$ 


\section{References}

Bates, D. (2005). Fitting linear mixed models in R. R news, 5(1), 27-30.

Bates, D., Mächler, M., Bolker, B., \& Walker, S. (2015). Fitting Linear Mixed-Effects Models Using lme4. Journal of Statistical Software, $67(1), 1-48$

Bolker, B. (2017). GLMM FAQ. Retrieved from http://bbolker.github.io/ mixedmodels-misc/glmmFAQ.html

Brennan, R. L. (2001a). Generalizability theory. New York, NY: Springer.

Brennan, R. L. (2001b). Manual for mGENOVA. Iowa City, IA: Iowa Testing Programs, University of Iowa.

Brooks, M. E., Kristensen, K., van Benthem, K. J., Magnusson, A., Berg, C. W., Nielsen, A., ... Bolker, B. M. (2017). glmmTMB balances speed and flexibility among packages for zero-inflated generalized linear mixed modeling. The R Journal, 9(2), 378-400.

Chen, Y., Dios, R., Mili, A., Wu, L., \& Wang, K. (2005). An empirical study of programming language trends. IEEE Software, 22(3), 7279.

Clauser, B. E., Harik, P., \& Margolis, M. J. (2006). A multivariate generalizability analysis of data from a performance assessment of physicians' clinical skills. Journal of Educational Measurement, 43(3), 173-191.

Cronbach, L. J., Rajaratnman, N., Gleser, G. C. (1963). Theory of generalizability: A liberalization of reliability theory. British Journal of Statistical Psychology, 16, 137-163.

Cronbach, L. J., Gleser, G. C., Nanda, H., \& Rajaratnam, N. (1972). The dependability of behavioral measurements: Theory of generalizability scores and profiles. New York, NY: Wiley.

R Development Core Team. (2019). R: A language and environment for statistical computing. R Foundation for Statistical Computing, Vienna, Austria. Retrieved from www.Rproject.org/

Doran, H., Bates, D., Bliese, P., \& Dowling, M. (2007). Estimating the multilevel Rasch model: With the lme4 package. Journal of Statistical software, 20(2), 1-18.

Geissmann, Q., Rodriguez, L. G., Beckwith, E. J., \& Gilestro, G. F. (2019). Rethomics: An R framework to analyse high-throughput behavioural data. PloS One, 14(1), e0209331.

Gleser, G., Cronbach, L., \& Rajaratnam, N. (1965). Generalizability of scores influenced by multiple sources of variance. Psychometrika, 30(4), 395-418.

Goodman, L. A. (1985). The analysis of cross-classified data having ordered and/or unordered categories: Association models, correlation models, and asymmetry models for contingency tables with or without missing entries. The Annals of Statistics, 13(1), 10-69.

Huebner, A., \& Lucht, M. (2019). Generalizability Theory in R. Practical Assessment, Research \& Evaluation, 24(5), 2.

Jiang, Z. (2018). Using linear mixed-effect model framework to estimate generalizability variance component in R: A lme4 package application. Methodology. 14(3), 133-142

Jiang, Z., \& Raymond, M. (2018). The use of multivariate generalizability theory to evaluate the quality of subscores. Applied Psychological Measurement, 42(8), 595-612.

Jiang, Z., \& Skorupski, W. (2018). A Bayesian approach to estimating variance components within a multivariate generalizability theory framework. Behavior Research Methods, 50(6), 2193-2214.

Jiang, Z., Walker, K., Shi, D., \& Cao, J. (2018). Improving generalizability coefficient estimate accuracy: A way to incorporate auxiliary information. Methodological Innovations, 11(2), 2059799118791397.

Joe, G. W., \& Woodward, J. A. (1976). Some developments in multivariate generalizability. Psychometrika, 41(2), 205-217.

Lance, C. E., LaPointe, J. A., \& Stewart, A. M. (1994). A test of the context dependency of three causal models of halo rater error. Journal of Applied Psychology, 79(3), 332.
Lander, J. P. (2014). R for everyone: advanced analytics and graphics. Pearson Education.

Li, Y., \& Baron, J. (2011). Behavioral research data analysis with $R$. Springer Science \& Business Media.

LoPilato, A. C., Carter, N. T., \& Wang, M. (2015). Updating generalizability theory in management research: Bayesian estimation of variance components. Journal of Management, 41(2), 692-717.

Lunn, D. J., Thomas, A., Best, N., \& Spiegelhalter, D. (2000). WinBUGS-a Bayesian modelling framework: concepts, structure, and extensibility. Statistics and Computing, 10(4), 325-337.

Magis, D., \& Barrada, J. R. (2017). Computerized adaptive testing with R: Recent updates of the package catR. Journal of Statistical Software, 76(1), 1-19.

Marcoulides, G. A. (1994). Selecting weighting schemes in multivariate generalizability studies. Educational and Psychological Measurement, 54(1), 3-7.

Marcoulides, G. A. (1995). Designing measurement studies under budget constraints: Controlling error of measurement and power. Educational and Psychological Measurement, 55 (3), 423-428.

Marcoulides, GA (1996) Estimating variance components in generalizability theory: The covariance structure analysis approach. Structural Equation Modeling 3: 290-299.

Moore, C. (2016). gtheory: Apply Generalizability Theory with $R$ [Computer software version]. Retrieved from https://cran.r-project. org/web/packages/gtheory/gtheory.pdf

$\mathrm{Nu} ß$ baum, A. (1984). Multivariate generalizability theory in educational measurement: An empirical study. Applied Psychological Measurement, 8, 219-230.

Plummer, M. (2003). JAGS: A program for analysis of Bayesian graphical models using Gibbs sampling. In K. Hornik, F. Leisch, \& A. Zeileis (Eds.), Proceedings of the 3rd International Workshop on Distributed Statistical Computing (Vol. 124, p. 125). Vienna, Austria: Technische Universität Wien. Retrieved from https:// www.r-project.org/conferences/DSC-2003/Proceedings/

Plummer, M. (2010). JAGS Version 2.2. 0 manual. Available from http:// mcmcjags. sourceforge.net.

Rao, P. S., \& Mudholkar, G. S. (1967). Generalized multivariate estimator for the mean of finite populations. Journal of the American Statistical Association, 62(319), 1009-1012.

Raudenbush, S. W., \& Bryk, A. S. (2002). Hierarchical linear models: Applications and data analysis methods (2nd). Thousand Oaks, CA: Sage.

Raykov, T., \& Marcoulides, G. A. (2006). Estimation of generalizability coefficients via a structural equation modeling approach to scale reliability evaluation. International Journal of Testing, 6(1), 81-95.

Raymond, M. R., \& Jiang, Z. (2019). Indices of Subscore Utility for Individuals and Subgroups Based on Multivariate Generalizability Theory. Educational and Psychological Measurement. Advance online publication. doi: https://doi.org/10.1177/0013164419846936.

Shavelson, R. J., \& Webb, N. M. (1981). Generalizability theory: 19731980. British Journal of Mathematical and Statistical Psychology, 34, 133-166.

Spiegelhalter, D. J., Thomas, A., Best, N. G., \& Lunn, D. (2003). WinBUGS user manual. Cambridge, UK: MRC Biostatistics Unit.

Srinivasan, V., \& Shocker, A. D. (1973). Estimating the weights for multiple attributes in a composite criterion using pairwise judgments. Psychometrika, 38(4), 473-493.

Stegmueller, D. (2013). How many countries for multilevel modeling? A comparison of frequentist and Bayesian approaches. American Journal of Political Science, 57(3), 748-761.

Thomas, A., O'Hara, B., Ligges, U., \& Sturtz, S. (2006). Making BUGS open. R News, 6(1), 12-17.

Wang \& Stanley (1970): Differential weighting: A review of methods and empirical studies. Review of Educational Research, 40, 663-705. 
Webb, N.M., Shavelson, R.J., \& Haertel, E.H. (2006). Reliability coefficients and generalizability theory. In C. R. Rao \& S. Sinharay Handbook of Statistics, 26 (pp. 1-124). Amsterdam: Elsevier.

Woodward, J. A., \& Joe, G. W. (1973). Maximizing the coefficient of generalizability in multi-facet decision studies. Psychometrika, 38(2), 173-181.
Wu, Y. F., \& Tzou, H. (2015). A multivariate generalizability theory approach to standard setting. Applied Psychological Measurement, $39(7), 507-524$.

Publisher's note Springer Nature remains neutral with regard to jurisdictional claims in published maps and institutional affiliations. 